\title{
LA UNIVERSIDAD COMO
} INSTITUCIÓN DE CONOCIMIENTO: ¿UNA DISCUSIÓN PENDIENTE EN CHILE?

Abelardo Castro* 


\section{RESUMEN}

Docencia, investigación y extensión, han sido consideradas, a través del tiempo, las tres funciones más esenciales de toda institución universitaria. Este artículo propone, mediante el examen histórico y comparativo, un modelo de la universidad como "Institución del Conocimiento", cuyas funciones serían la generación, transferencia y gestión del mismo. Analiza la manera como se están dando estas funciones en las universidades chilenas y avanza hacia cómo debieran darse en una sociedad como la actual, caracterizada por la globalidad y el predominio del mercado. Muestra las inconsecuencias que se dan en el actual esquema de incentivo y financiamiento en Chile, particularmente las que tienen efecto en el rol de la universidad en la sociedad, y apunta a algunas soluciones que podrían producir el tipo de universidad que queremos para el país.

\section{ABSTRACT}

Teaching, research and outreach have been considered, through time, the three essential activities of any university. This paper proposes, through a historical and comparative analysis, a model of universities as the "Institution of Knowledge". Their functions would be to generate, transfer and manage knowledge. The paper analyzes the way in which these functions are being conducted in Chilean universities and proposes how they should be performed in a society with features such as the current one, characterized by globalization and a predominance of the market. It highlights the inconsistencies evident in the current scheme of incentives and financing in Chile, particularly in those that have an effect on the role of universities in society and it points to some solutions that could produce the kind of university that we would like to have in our country. 


\section{LA UNIVERSIDAD COMO INSTITUCIÓN DE CONOCIMIENTO: ¿UNA DISCUSIÓN PENDIENTE EN CHILE?}

El tema de la universidad emerge cada vez con mayor fuerza, tal como ha ocurrido en época de transiciones históricas, a través de su existencia. Hoy vivenciamos cambios que eran inimaginables hace algunas décadas y, por lo mismo, cabe replantearse la pregunta acerca del norte y los desafíos de la universidad.

La universidad en la sociedad posmoderna

Surgidas en la Edad Media, ya sea como corporaciones de alumnos y maestros o de maestros y alumnos, las universidades han enfrentado diversos desafíos, debiendo sortear y adaptarse a situaciones tan variadas como la supremacía de la Iglesia y el surgimiento de los estados nacionales, cada uno con expectativas socioculturales distintas. De acuerdo a las circunstancias específicas, encontramos diversidad de contextos en que ellas actúan y formas de acción que adoptan frente a esos contextos. Así, por ejemplo, en la actualidad, las universidades europeas trabajan protegidas y reguladas por el Estado. En Estados Unidos y América Latina coexisten universidades privadas y públicas que compiten entre sí por recursos privados y públicos, ofreciendo formación profesional, asistencia técnica e investigación a distintos actores sociales, como alumnos empresas o Estados.

Un mundo globalizado

En nuestro análisis no puede pasar inadvertido el hecho histórico social de vivir en lo que se ha denominado "la aldea global", en el contexto 
de economías de libre mercado. Cambios importantes en la informática y en los sistemas de transporte han provocado también transformaciones profundas en los procesos productivos y de servicios y, por ende, en la interacción humana. El mundo se ha empequeñecido, empresas, capitales y conocimientos se transfieren de un lugar a otro en plazos breves. La movilidad, capacidad de percibir el cambio y actuar con precisión son factores esenciales del éxito y sobrevivencia en un mundo globalizado, que reconoce la economía de libre mercado como axioma central de la actividad económica, incluidas las universidades.

En esta lógica, éstas también han ingresado a la "disputa por mercados" no sólo dentro de los estados donde operan, sino, también, fuera de ellos. No deja de ser sintomático, por ejemplo, que la Universidad de Harvard dicte cursos en administración en Santiago y se apreste a hacerlo también en el área de Educación.

\section{Diversificación interna}

La necesidad de atraer y competir por recursos -que no son de fácil acceso- ha generado una tendencia a la "especialización" de funciones académicas o a transformar la universidad en una "multiversidad". Daniel Alpert, resume el fenómeno: "En años recientes ha existido una fuerte tendencia hacia una fragmentación mayor de la organización -con frecuencia en el ámbito del profesor individual. El proceso se ha acelerado por varios hechos [...] entre ellos el aumento permanente de la especialización, el sistema para evaluar el rendimiento y los mecanismos que se emplean para contratar los apoyos federales a la investigación académica (Alpert, 1993)”.

La investigación y la docencia, que, en esencia, eran partes del todo académico que constituía la excelencia docente, han tendido a disgregarse. La especialización llegó a tal extremo que, actualmente, se establecen clasificaciones tales como universidades docentes y universidades de investigación, con los respectivos efectos colaterales. Estas últimas enfrentan hoy un dilema no fácil de resolver, puesto que los recursos de investigación no son suficientes para autofinanciarse. La universidad docente puede hacerlo, pero necesita de la investigación para perfilar la oferta. 
Cambios en la manera de ver la universidad posmoderna

Desde la perspectiva tradicional, gran parte de la discusión se ha centrado en las funciones de la universidad y, en otros casos, en la misión de la misma. Ocurre en el último caso que el análisis se confunde con las funciones y misión de la ciencia en la sociedad.

Cuando se abordaba el tema de las funciones de la universidad, solía, y suele, decirse que éstas son fundamentalmente tres: investigación, docencia y extensión. Se han agregado a ellas la asistencia técnica y la educación continua. Incluso puede haber otras, por ejemplo: difusión cultural. La "tentación" mayor de este tipo de aproximación es la facilidad con que cada una de ellas pueda analizarse con independencia de la otra y, desde el punto de vista de la estructura universitaria, crearse organismos separados y "autónomos" que aborden cada una de estas expresiones de universidad.

Los enfoques que tienen como aproximación la misión de la universidad extrapolan a su análisis la discusión de la independencia o dependencia de la ciencia respecto a la sociedad y su sitial en ella. Max Weber plantea, por ejemplo, que la misión de la ciencia es desmitificar la realidad; por tanto, debe tener autonomía en relación con presiones externas. En cuanto a la universidad, lugar natural de la ciencia, señala que su misión radica en la generación del conocimiento (cuya función es desmitificar la realidad) y la formación de científicos (Weber, 1977).

Visión distinta a la anterior constituye la propuesta de la universidad "comprometida" con el cambio social y el desarrollo del país, que toma fuerza en América Latina a partir del llamado Movimiento de Córdoba. Se espera que la universidad "responda" a las necesidades del país a través de la formación de profesionales, investigación relevante -desde el punto de vista del desarrollo socioeconómico fundamentalmente-y difusión del saber (Tünnerman, 1970; Castro y Kalpazidou, 1985).

En un intento por hacer converger visiones y opciones de universidad, está la idea de percibirla desde el punto de vista de su misión relacionada con la ciencia y la administración eficiente de la 
misma, para provocar efectos deseables en la sociedad. Así, se parte de la premisa de que la universidad es una Institución de Conocimiento. Su misión es trabajar con el conocimiento, la que toma distintas expresiones circunstanciales dependiendo del momento en que se encuentre el "estado del arte" en una área específica. A partir de esta consideración se expresa que, en cuanto "función" (expresión circunstancial del trabajo con el conocimiento), estas expresiones son fundamentalmente tres: a) generación, b) transferencia y c) gestión del conocimiento. Se asume que el saber científico tiene un efecto desmitificador e impacto social dependiendo de cómo se gestione el mismo.

Generación de conocimiento: Este concepto es, efectivamente, de un alcance mayor al de "investigación científica", usado tradicionalmente. Se parte del supuesto que el conocimiento se obtiene por formas distintas a la experimentación y observación directa, y se acepta la idea de "experiencia probada" para indicar la generación de conocimientos tecnológicos para el cual no siempre existe explicación.

Transferencia de conocimiento: Comprende más que la docencia organizada en programas de formación profesional heredados del modelo napoleónico de "escuelas superiores". Es más, se enfatiza que la transferencia de conocimiento implica dos momentos esenciales: a) conocimiento logrado, expresado en forma de texto, archivos, entre otros, y b) el proceso a través del cual éste es generado.

Gestión del conocimiento: Los dos aspectos mencionados anteriormente han estado presentes en toda la discusión tradicional acerca de la universidad. No es el caso del tema de la gestión. A pesar de estar extremadamente presente en el hacer universidad, no se ha abordado mayormente como categoría de análisis, sino en los últimos años. Hoy parece un tema de moda.

Mientras algunas universidades optaban por modelos como el diseñado por Humboldt, el tema de la formación de profesionales parecía ser secundario. El estudiante "armaba", o arma, su profesión de forma activa, considerando las líneas de investigación y ciencias existentes en una universidad. En las escuelas superiores del modelo napoleónico predominaron la formación profesional y la investigación, determinadas por las necesidades de las carreras. 
En ambos casos se han producido cambios que, a su vez, generan otras interrogantes. En el primero, se dio la tendencia a estructurar en forma más consistente las profesiones; en el segundo, la investigación se ha hecho más independiente de las necesidades de la profesión.

El hecho de considerar el conocimiento como base de la actividad universitaria, y del cual se derivan actividades concretas como el surgimiento de nuevas profesiones, plantea, por una parte, cómo coordinar las mismas y, por otra, cómo salvaguardar el principio de sinergia en el sentido de que, siendo actividades distintas, se potencien y enriquezcan mutuamente (Dahllöf, 1997).

Teóricamente, unir la docencia con la investigación no es difícil; menos cuando se trata de la docencia de posgrado. La transferencia de conocimiento hacia fuera de la universidad, o extensión universitaria, es mucho más compleja.

En circunstancias actuales, el investigador no puede trabajar solo, necesita un equipo que lo acompañe; pueden ser otros colegas o, como ocurre usualmente, sus alumnos de posgrado. Si se une de manera eficiente la educación de posgrado con la investigación científica, es posible incrementar sustancialmente la generación de conocimientos. En ese sentido, la universidad está cumpliendo la función de desmitificación que señala Weber, y la contribución a la sociedad, por gracia, que plantea Humboldt, en tanto se forman nuevos talentos científicos con posibilidad de aplicar el conocimiento adquirido (Gustavsson, 1971). Sin embargo, el aporte ha circulado todavía en relación con la generación de conocimiento. Con el fin de que el conocimiento llegue de forma útil a la sociedad se requiere de la formación de profesionales en distintas áreas del saber, actividad que se ha denominado "pregrado". Esta forma de transferencia de conocimiento es la más estructurada de las actividades universitarias.

Debido a las características que ha alcanzado el desarrollo social, han surgido nuevas expectativas relacionadas con este grado de formación. A la erudición de orientación práctica se agrega, como exigencia, un conjunto de habilidades de orden cognitivo como, por ejemplo, la resolución de problemas. Esta exigencia intenta resolver, modificando las formas del ejercicio de la docencia y vinculándola a 
un fenómeno propio de la investigación, el proceso a través del cual se genera el saber científico, como la pedagogía basada en la resolución de problemas, la formación por investigación, por proyecto y otras. Se trata de formar profesionales en conocimientos adquiridos y procesos de la ciencia.

La asociación de la gestión con el conocimiento implica, además, considerar el tema del financiamiento de la actividad universitaria. Como la mayoría de las actividades humanas, el tema de los recursos es determinante para el crecimiento y fortalecimiento de la actividad universitaria.

Desde ese punto de vista, se hace aún más necesario producir el principio de sinergia entre las distintas formas de expresión del trabajo universitario. Al equilibrio de funciones debe sumarse el equilibrio presupuestario.

El rol de la economía tuvo un papel central en los primeros modelos de universidad. Bolonia, por ejemplo, nació como una Corporación de Alumnos (universitas scholarum); eran alumnos que se agrupaban en torno a eruditos de la época. En el caso de la Universidad de La Sorbone en París, se trataba de una Corporación de Maestros (universitas magistrorum). En ambas eran los alumnos quienes financiaban la enseñanza, sin embargo, en el primero, los profesores estaban "en manos" de los alumnos. Buscando protegerse de lo mismo, los parisinos se asociaron para resguardar sus derechos y dar estabilidad a su actividad.

Con el surgimiento de los estados nacionales, nació el aporte público como otra fuente de financiamiento trayendo también otras exigencias a la educación superior. En ese contexto han surgido nuevas necesidades de equilibrio que deben ser consideradas en la gestión universitaria. Por una parte, el interés de los actores (alumnos y maestros) y, por otra, el interés social representado por el Estado y sus políticas financieras de la educación superior, las que no siempre ayudan al equilibrio interno del desarrollo del saber científico y tecnológico en las universidades, afectando de paso los equilibrios en el sistema de remuneraciones y carrera académica del personal docente. 
Aproximación a las universidades chilenas

Transferencia de conocimientos

Si miramos el amplio espectro de la educación superior en Chile y lo comparamos con la situación contextual y los modelos de los países más desarrollados, encontramos una serie de disparidades que llama profundamente la atención.

Desde el punto de vista de la organización de la docencia, nos encontramos con que nuestras universidades más parecen liceos para adolescentes mayores que universidades. El trabajo independiente de aproximación al conocimiento y la creación artística tiene, en las universidades chilenas, un espacio muy reducido. Mientras un alumno de ciencias sociales de una universidad sueca está obligado a leer, como mínimo, un libro por semana, nuestros alumnos acuden a las aulas a tomar apuntes y aprender de lo que expone el profesor. En el mejor de los casos, se realizan trabajos en grupos, donde la lectura intensiva se concentra en capítulos de libros. Este hecho no sólo afecta la cantidad de conocimientos que deben ser incorporados al egresado, sino que, además, afecta la capacidad de resolución de problemas y la creatividad del futuro profesional, frenando su desarrollo pleno.

La formación, en general, se lleva cabo en un esquema de distribución del tiempo pedagógico en donde los estudiantes tienen entre seis y ocho asignaturas paralelas. La consecuencia lógica de ello es que el aprendizaje logrado es de tipo superficial, se "picotea" una y otra disciplina sin profundizar ni incrementar estrategias de aprendizaje de tipo profundo. Para aprobar, el estudiante se transforma en experto en sobrevivencia académica, descuidando conscientemente, a fin de semestre, las asignaturas en que le fue mejor en el primer certamen para preocuparse de aquellas en que su rendimiento fue deficiente.

Este esquema de distribución del tiempo pedagógico, que tiene su origen en concepciones de aprendizaje memorístico de finales del 1800 , no es menos perjudicial para el desarrollo del académico. Atado a la docencia, encuentra dificultades para tener espacios para la reflexión, actualización y generación de nuevos conocimientos. El intercambio de experiencia y pasantías en otros lugares está supeditado 
a que alguien reemplace su presencia en clases coartando así su posibilidad de construir redes de conocimiento, factor clave en esta sociedad globalizada. Finalmente, este concepto de docencia lleva consigo otra dificultad: al profesor universitario se le considera su docencia como horas presenciales frente al alumno, como si su actividad fuera similar a la de un tornero o albañil. A consecuencia de ello, el profesor universitario se ve forzado a defender sus horas de clases y la presencia de su asignatura, se siente obligado a recargarse de trabajo y participar en la generación de mallas repletas de asignaturas que él mismo, en su interior, siente que no son buenas, ni para él, en términos de desarrollo de excelencia académica, ni para el alumno, en lo concerniente a la adquisición de competencias claves.

Nuestros alumnos han cambiado. La misma tecnología que hace posible el crecimiento exponencial del conocimiento ha generado condiciones que están influyendo en las características del estudiantado y en sus condiciones de trabajo. El monopolio de la transferencia del conocimiento por parte de las instituciones que históricamente lo hicieron se ha quebrado. Las fuentes de las cuales los estudiantes pueden recibir la información científica y profesional son muy diversas y de alta eficiencia. Esto determina que su relación con los profesores y sus pares sea distinta, el docente no tiene el "monopolio" del saber y, por esto, el alumno está en mejores condiciones para desarrollar diálogos académicos con sus maestros en un plano de mayor equidad. La información que cada uno de ellos adquiere es muy diversa en contenidos y estructuras; por lo mismo, la relación con los pares ha cambiado: hay mayor variedad de opiniones y tendencias de pensamiento.

Los cambios en el área de la informática, el acceso más libre al conocimiento hace posible, además, que los alumnos alcancen niveles de excelencia impensables algunos años atrás, donde el tomar apuntes era la forma esencial de aprender. El mundo de los jóvenes ya no es lo próximo, como la ciudad o el país en que crecieron: es el planeta, el universo.

En ese sentido, no deja de ser interesante el modelo de docencia practicado con frecuencia en las universidades nórdicas, donde las asignaturas se dictan de una o dos simultáneamente, como módulos 
de aprendizaje. Esto permite al alumno profundizar uno o dos temas a la vez, mientras el profesor de tiempo completo -entre uno y otro módulo de su responsabilidad-puede dedicarse a su perfeccionamiento in situ o fuera de su país, cuando existen los recursos para ello. Este sistema centra su atención en el desarrollo del docente a fin de que la calidad de su trabajo impregne la formación profesional o la investigación.

El acceso a la informática se está masificando, ofreciendo cada vez nuevas oportunidades. La universidad debe, con audacia y asentada en los estilos tradicionales de formación, pasar a procesos con apoyo en la virtualidad. Se debe combinar la forma presencial con las aulas virtuales y la conferencia con el estudio individual, todo ello en programas de estudios flexibles que respondan a necesidades diversas de públicos también diversos.

Por alguna razón, no siempre científica, las universidades se transformaron en instituciones de certificación de carrera de grado superior. Si alguna persona requiere un curso, de meses o un semestre de duración y respecto a un tema puntual, no tiene adonde acudir. Esto en un mundo que requiere flexibilidad laboral y formación permanente. ¿Qué ocurre con los resultados de la investigación en un área? ¿Se debe esperar la implementación de carreras para poder acceder a ellos? La impresión es que se hace urgente abrir la universidad a quienes quieran realizar estudios libres, en temas puntuales y en una de las áreas de excelencia de la universidad, sin que ello signifique tener que participar en paquetes cerrados de formación. Esos cursos libres pueden llegar a ser homologables en el contexto de alguna carrera o programa, en una perspectiva de educación permanente.

El Ministerio de Educación de Chile ha hecho explícita la formación en los llamados objetivos transversales de la educación, en temas tales como el cuidado del medio ambiente, la solidaridad, el desarrollo de habilidades intelectuales de orden superior, el desarrollo de proyectos de vida, entre otros. Esos objetivos se alcanzan a través de la información que imparte el docente, pero están fundamentalmente relacionados con el cómo se estructura el proceso educativo, con la metodología de la enseñanza. Se trata de objetivos 
necesarios para que un ciudadano pueda desarrollarse en la sociedad actual y que la universidad debe recoger $\left(\mathrm{CINDA}^{1}, 2000\right)$. Y, para quien piensa en costos, la inclusión de esos objetivos y su ejecución a través de los procesos de formación no implica mayores insumos que la educación frontal.

En un intento por incorporar esa transversalidad en la formación profesional, tanto la Universidad de Chile como la Pontificia Universidad Católica de Chile se encuentran conformando un plan de bachillerato al interior de las carreras, medida que no deja de ser interesante.

Generación de conocimientos y de creación artística

A fines de la década del sesenta, y con el objetivo de impulsar el desarrollo científico, se creó la Comisión Nacional de Investigación Científica y Tecnológica (CONICYT) y, en lo que respecta al arte, este último tiempo, el Fondo Nacional de Desarrollo Cultural y de las Artes (Fondart). Al interior de las universidades, se crearon direcciones homólogas con el mismo objetivo. Obviamente que el impacto de estas instituciones, en lo que a generación de conocimientos se refiere, ha tenido consecuencias positivas. Sin embargo, no puede impedir los efectos colaterales y negativos en la vida universitaria. Al interior de las universidades existen investigaciones científicas reconocidas y no reconocidas, unas que cuentan con recursos -y el docente puede dedicar tiempo universitario-y otras que se ejecutan quitando horas al tiempo libre y familiar. Están, además, por una parte, las investigaciones que contribuyen al desarrollo del académico en términos de ascensos y aumento de remuneraciones, y, por otra, aquellas que por no ser el Fondo Nacional de Desarrollo Científico y Tecnológico (Fondecyt) o cualquier otro "Fond" no tienen mayor valor de mérito oficial y resulta difícil ingresarla a la contabilidad del mérito académico. En algunos casos, la situación se complica cuando la investigación tiene asignación horaria que excluye de la docencia sin que los resultados lleguen o, de hacerlo, esto ocurra en forma muy diluida. En esos casos se produce, entonces, una barrera casi infranqueable entre la investigación y la docencia que no es deseable.

1 Centro Interuniversitario de Desarrollo. 
Si se aplica la lógica en que se desenvuelve una empresa de alta tecnología, expuesta a las leyes del mercado, las universidades ya estarían quebradas hace mucho tiempo. El principio básico de estas empresas es hacer llegar los resultados científicos lo más rápido posible al proceso productivo y al mercado. Su ventaja comparativa radica en cuán eficientemente se actúa en ese proceso. Esta lógica de interacción es casi desconocida en el mundo de la educación superior en Chile. Y las universidades representadas en los "Fond", con altos porcentajes de investigación, no siempre aprovechan las ventajas que les da generar conocimiento. Una lista de las carreras ofrecidas así lo demuestra; no hay carreras nuevas o cursos nuevos en los cuales se expresen los resultados de esas investigaciones: son muy pocos.

En el afán por impulsar la capacidad de investigación, y considerando la escasez de recursos para esta actividad, se asumió la modalidad de fondos concursables vía presentación de proyectos de investigación. La evaluación de los mismos recae en pares externos a la universidad, los que, por cierto, no conocen las líneas prioritarias de investigación de una facultad determinada, ni tampoco las áreas en que éstas pretenden perfilarse. Constituyendo estos fondos concursables la mayor parte de los recursos destinados a la investigación, es fácil imaginar lo que ocurre con la investigación al interior de la facultades y departamentos. La política de investigación es guiada desde fuera, de acuerdo a los vaivenes y gustos de los evaluadores externos. Así, la investigación universitaria es como la posmodernidad misma: está llena de incertidumbre y no se sabe exactamente hacia donde va o hacia donde nos lleva.

Esta situación solamente es reversible incrementando los gastos fijos en investigación y dejando los fondos concursables como complemento o líneas nuevas de investigación o refuerzo de las ya existentes. Las universidades europeas han resuelto este problema asociando la investigación a la carga regular del docente y, en especial, a los professor que en algunos países disponen de hasta el $60 \%$ de tiempo académico a la investigación y a la dirección de tesis de doctorado. Asimismo, a estos professor, que además generan recursos a través de nuevas líneas de investigación, les depara un retiro digno, en el sentido de que la institución los sigue apoyando en la búsqueda 
de recursos, manteniendo también las condiciones de trabajo adecuadas a "viejo" profesor.

La conexión "posgrado investigación" no deja de ser interesante. Difícilmente las universidades pueden cubrir las distintas áreas de desarrollo dentro de una disciplina a través de sus académicos de planta, encontrándose la solución en los programas de doctorado. Los recursos externos son, en general, ocupados en estipendios, en doctorandos que, por una parte, integran un equipo de investigación y, por otra, hacen clases en el ámbito de pregrado. De esta manera se garantiza la estabilidad en las líneas de investigación y la llegada de contenidos actualizados y de alto nivel a los alumnos de formación universitaria inicial.

\section{Gestión del conocimiento}

En las tradiciones anglosajona, francesa y nórdica encontramos una diferencia entre lo que podríamos llamar universidades docentes y universidades de investigación (college - university - école - universida, högskolor - universitet). Sin embargo, si en la actualidad observamos esas "tradiciones", nos daremos cuenta de que la división entre ambas no es en absoluto tan tajante. Así la École Central de París, creada bajo el concepto napoleónico, se ha transformado en uno de los centros tecnológicos de investigación más importantes de Francia. Lo mismo ocurre con la Kungliga Tekniska Högskola de Estocolmo, que presenta un fuerte desarrollo del posgrado. Al revés, muchas universidades, cuyo centro era la investigación, no han perdido el pregrado y han pasado a ofrecer también carreras profesionales.

Este hecho nos lleva a pensar que, en realidad, no es necesario optar entre universidades docentes y de investigación. Esa dicotomía, en términos absolutos casi no existe; sí es posible acentuar una u otra alternativa. Si una institución de educación superior opta por permanecer en el tiempo, requiere de ambas actividades, y por cierto que la que mejor integre ambas es la que estará mejor posicionada, al igual que ocurre con las empresas de alta tecnología: mientras mejor integrada esté la investigación a la producción y las necesidades de corto y largo plazo del mercado, mayor probabilidad de subsistencia en el tiempo y de expansión. 
Por lo anterior, no deja de ser preocupante el tema de la gestión del conocimiento y las estructuras para posibilitar su desarrollo y transferencia a la sociedad que lo demanda, cuando se estudian las universidades chilenas. Su administración sigue siendo la de una institución pública que debía formar los profesionales que necesitaba un estado naciente, en circunstancias que las condiciones han cambiado radicalmente. Ha pasado el tiempo en que las universidades tradicionales tenían el monopolio de la certificación de formación superior. El ingreso de las instituciones privadas a este ámbito ha cambiado el escenario de la formación de profesionales de manera radical. A la ofensiva y agresividad con que ellas ingresan a este nivel de actividad, las universidades tradicionales responden con protestas, desdeños o solicitando recursos al Estado. Si las instituciones privadas son "exitosas" ofreciendo la misma formación que las universidades tradicionales, cabe preguntarse: ¿cómo se explica su nivel de expansión? Las respuestas no hay que buscarlas sólo en el "mercado del conocimiento", sino, como ocurre en la industria de alta tecnología, en la capacidad de gestión propia. Si otras universidades se han expandido haciéndolo con el recurso humano de las universidades tradicionales es una muestra más de la crisis profunda de estas instituciones tradicionales: significa que no han sido lo suficientemente dinámicas en su desarrollo y tampoco han tenido una política personal que estimule la identificación plena de los docentes con la institución.

\section{Los nuevos desafíos}

Hasta el momento se ha planteado que la preocupación básica de las universidades es el conocimiento (incluyendo la creación artística), su generación, transferencia y gestión, de forma coherente. Son pocos quienes podrían discrepar de esta caracterización. Tampoco es posible negar la celeridad con que se están publicando resultados científicos, ni tampoco los avasalladores cambios producidos en el mundo productivo y de servicios. Esto ha hecho pensar a muchos que la entrega eficiente de conocimientos ya no basta; no es suficiente para que el individuo se enfrente como profesional a la sociedad y sus requerimientos. Las reformas educacionales, en las más diversas partes del mundo, han constatado este hecho y lo han asumido incorporando nuevos elementos a sus planes de estudios: los "objetivos transversales". Entre ellos está la necesidad de desarrollar capacidades 
cognitivas de orden superior, la aproximación independiente al conocimiento, la capacidad de resolución de problemas y la innovación, todos aspectos considerados claves. Tomará unos años antes de que estos planes funcionen en la enseñanza media. Pero, ¿qué ocurrirá cuando alumnos con otra formación a la que tienen los que ingresan actualmente lleguen a la educación superior tradicional? ¿Qué pasará con las postulaciones de alumnos conscientes de que el mundo laboral les exige esas competencias como mínimo, porque también están los transversales de tipo social e individual? Si se observa con atención a quienes contrata la industria de alta tecnología, la que vive de la generación de conocimientos - al igual que las universidades- se puede constatar que no se trata de personas con un alto nivel de conocimientos, sino que, además de lo anterior, son capaces de demostrar un gran desarrollo de procesos intelectuales complejos. Este es un hecho del que muy pocas universidades se han hecho cargo.

Para el caso de la gestión universitaria es este un tema interesante. Las universidades de países desarrollados han planteado que el problema se resuelve "enseñando en el espíritu de la ciencia", vale decir: una buena formación en los resultados obtenidos por la ciencia y sometiendo a sus alumnos al proceso de investigación por mínimo que estos esfuerzos sean. Esto lo realizan, en muchos casos, con un número superior de alumnos por profesor -cuyo esfuerzo medido en horas frente al alumno es menor- y con mejor remuneración y clima de trabajo más satisfactorio.

Lo viejo y nuevo de las visiones respecto al ser de la universidad

En la actualidad, y por directrices del Ministerio de Educación, las universidades subvencionadas deben tener un plan de desarrollo estratégico, con una visión de lo que se espera de la institución en un plazo predecible. Los componentes fundamentales de una visión es el "estado del arte" respecto de lo que se quiere hacer y el componente valórico. En ese sentido no deja de llamar la atención que la visión expuesta en los planes estratégicos no sea más que pronóstico de metas a cumplir, relacionadas con lo que ya se está haciendo. Es decir, más de lo mismo, pero en un mundo distinto. Es verdad que muchos de los planes estratégicos presentados por las universidades tradicionales al Ministerio de Educación fueron elaborados para cumplir con un 
requisito administrativo que les permitiera acceder a recursos frescos. Sin embargo, la realidad está mostrando que eso no corresponde. Menos aún que el tema de compromiso de las universidades con la sociedad que las cofinancia se reduzca a frases dictadas por la necesidad financiera.

Un tema final

Otra diferencia de la aldea global con épocas anteriores es el desarrollo de formas "blandas" de producción y de servicios. En ese nuevo contexto, la mujer, que se ha incorporado al trabajo fuera del hogar, con características intelectuales particulares y complementarias a las del hombre, irrumpe también con fuerza en el mundo académico. Igualmente un signo posmoderno. Pero esta incorporación al mundo académico no ha sido fácil. Si los jóvenes han sentido con fuerza la vivencia de no ser escuchados, tanto o más grande ha sido el sentimiento de las académicas de nuestras universidades, pues son escasos los cargos en que ellas se encuentran representadas. Si hiciéramos caso a los aportes científicos que no hablan de inteligencias múltiples e inteligencias emocionales, no nos cabría duda que en la conformación de los equipos directivos deberían estar presentes. Esto no es así. Creo que este es también un desafío posmoderno. 


\section{ReFERENCIAS BibliográficAS}

Alpert, D., "Rethinking the challenges facing the American research university", Unpublished manuscript, en Awbrey SM, Scott DK, The Third Transformation Universities into the Next Century, disponible en http://www.umass.edu/pastchancellors/scott/papers/transform.html Acceso en junio de 2004.

Castro, A. y Kalpazidou, E., Uppfattningar om forskning och samhälle. Pedagogiska Institutionen, Uppsala Universitet, Uppsala, 1985.

CINDA 2000, Las Nuevas Demandas del Desempeño Profesional y sus Implicancias para la Docencia Universitaria, Santiago, Chile, 2000.

Dahllöf, U., Tertiary Education and Regional Development in Cross National Perspective, CHEMP, University of New England, New England, Australia, 1999.

Gustavsson, S, Debatten om forskningen och samhället, Stockholm: Almqvist \& Wiksell, 1971.

Nybom, T., Universitet och Samhälle: Om forsknings-politik och vetenskapens samhälleliga roll, Tiden, Stockholm, 1989.

Tünnerman, Carlos, La reforma universitaria de Córdoba, FEDES, Caracas, 1970.

Weber, M, Vetenskap och politik, Göteborg: Korpen, 1977. 